\title{
Dying patients with COVID-19: What should Hospital Palliative Care Teams (HPCTs) be prepared for?
}

\section{Case Report}

Cite this article: Anneser J (2020). Dying patients with COVID-19: What should Hospital Palliative Care Teams (HPCTs) be prepared for? Palliative and Supportive Care 18, 382-384. https://doi.org/10.1017/S1478951520000450

Received: 1 April 2020

Revised: 9 June 2020

Accepted: 10 June 2020

\section{Key words:}

COVID-19; Hospital Palliative Care Team; Symptom control; Visiting restrictions

Author for correspondence: Johanna Anneser, Department of Psychosomatic Medicine and Psychotherapy, Klinikum rechts der Isar, Technical University of Munich, Ismaninger Str. 22, München 81664, Germany. E-mail: j.anneser@tum.de
Johanna Anneser, PROFESSOR, M.D. (D)

Palliative Care Team, Department of Psychosomatic Medicine and Psychotherapy, School of Medicine, Technical University of Munich, Munich, Germany

\begin{abstract}
Objective. The COVID-19 pandemic is a care crisis of unknown duration which has seemingly not yet reached its peak in many countries. A significant number of elderly and frail people and those with underlying serious illness will continue to develop severe forms of the COVID-19 infection. Most of them are not eligible for intensive care treatment but can still expect palliative care - in many cases provided by a Hospital Palliative Care Team (HPCT). Several teams have already gained experience in caring for these patients and their families, others are preparing for it.

Method. We report on a COVID-19 patient with pre-existing acute myeloid leukemia who was looked after by a HPCT until death. We discuss the challenges and difficulties while caring for COVID-19-positive palliative patients in a non-ICU setting.

Results. Hospitalization of the patient in an isolation ward caused an enormous burden for the dying patient and his family. Symptom control was particularly difficult because of rapid deteriorating dyspnea and the scarce presence of medical staff in the patient's room.

Significance of results. COVID-19 patients who are not eligible for ICU treatment may have a particularly high need for palliative care. Since beds in specialist palliative care units are limited, the HPCT should be prepared to care for these patients. They may offer support in decision-making, optimize symptom control, and provide psychosocial care for patients and their families. Visiting restrictions aimed to protect the general public must be weighted against the patient's and family's suffering.
\end{abstract}

\section{Introduction}

While, in many countries, the initial pandemic wave approaches its peak, others expect its resurgence (Kissler et al., 2020). Some patients affected by COVID-19 will continue to die in specialist palliative care units, a considerable number in ICUs, very many in ordinary wards, and even more in nursing homes. Beds in palliative care units are limited and are not available for all patients who are in need of it. Intensive care treatment may not be wanted, not indicated, or not possible because of the lack of available resources. Providing care in an ordinary ward as result of a triage decision is extremely challenging as we face the fears and anger of patients and their families because access to a potential live-saving therapy has been denied. It has to kept in mind that one of the key principles of the triage process is that all patients will be cared for in the best possible way (Downar et al., 2010). This unquestionably includes access to palliative care (Borasio et al., 2020) that is to a significant extent provided by HPCTs.

We describe a cancer patient affected by COVID-19 in a German tertiary hospital who has been followed by a HPCT. Based on the case report, we discuss challenges and difficulties HPCTs may face when treating a palliative COVID-19 patient during the pandemic.

\section{Case report}

A 74-year-old male presented to the emergency department with fatigue, weakness, dyspnea, and melena for several days. Seven years before, the patient had been diagnosed with acute myeloid leukemia and had been treated with several chemotherapy regimens. On admission, the patient was afebrile and presented with mild cough and increased mucous phlegm. $\mathrm{He}$ received erythrocyte and platelet concentrates since laboratory findings included anemia $(2.3 \mathrm{~T} / \mathrm{l})$ and thrombocytopenia $(52 \mathrm{G} / \mathrm{l})$. On the same day, the patient's nasopharyngeal and oropharyngeal swabs were tested positive for COVID-19 and he was transferred to an isolation ward. After a phase of stabilization, he developed respiratory and renal failure. Due to the pre-existing severe underlying disease, intubation and intensive care treatment did not seem promising, and the hospital's HPCT was involved in the patient's care. His main physical 
complaints three days prior to his death were dyspnea and weakness. He repeatedly expressed his distress that no visits were allowed. Even though he was able to communicate via his mobile phone, he felt profoundly lonely and cut off from his family. As a single exception during a period of eight days, his daughter was allowed to visit him once for $30 \mathrm{~min}$. Two days before his death, his dyspnea worsened and he had an increasing demand for oxygen. Intravenous treatment with morphine was started and had to be adjusted frequently. Over a period of $12 \mathrm{~h}$, the patient became comatose and died 11 days after admission.

\section{Discussion}

\section{Advance care planning and discussing goals of care}

HPCTs provide inpatients from all departments palliative medical support. Seriously ill as well as dying patients, who are being treated in facilities that do not have their own palliative care unit, can benefit from these multiprofessional teams just as much as patients who do not (yet) need to be admitted to a palliative care unit. In addition, many specialized palliative care units are not able to offer treatment to COVID-19-positive patients. HCPTs are often well integrated into the clinical processes of various departments and may help to clarify goals of care in time. Advance care planning in patients at high risk for severe disease may often result in do-not-intubate orders. This will considerably ease the burden on the health system and make resources available for other patients. Other patients may not be eligible for intensive care treatment - even if they wish to be treated in an ICU - because of the lack of a medical indication, for example patients with an underlying life-limiting, rapidly progressing disease. Well before the pandemic reaches its peak or before the next pandemic wave, HCPTs should proactively facilitate discussions regarding medical indications for ICU treatment, if possible also in patients who are not yet followed by the palliative care team.

\section{The role of HCPTs in triage decisions}

In the past, the COVID-19 pandemic has already led to shortages in personal protective equipments such as face masks and protective clothing. It is foreseeable that in many countries, ICU beds and ventilators will still not be sufficiently available and triage decisions will be made (Truog et al., 2020). Various medical societies have published frameworks for such decisions (Emanuel et al., 2020; Swiss Academy of Medical Sciences, 2020). As stated before (Borasio et al., 2020), it is not the scope nor the competence of palliative care specialists to use different triage criteria, but in this process, HPCTs should ensure that the patients, who are not eligible for ICU treatment, will receive adequate medication for symptom control and help to prevent that patients and their families feel abandoned.

\section{Symptom control}

Many physicians and nurses in ordinary wards may be unfamiliar with the treatment of dying patients, and their workload is immense during a pandemic wave. Along with the need to put on personal protective equipment when entering the patient's room and the risk to contract the virus, visits from the medical staff are often kept to a minimum. On the other hand, the respiratory function of patients affected by COVID-19 can deteriorate rapidly (Goh et al., 2020) and frequent adjustments of medication may be necessary. To ensure optimal symptom control, standardized order sheets and protocols with precise instructions should be in place, so that adjustments can be made as timely as possible.

\section{Psychosocial and spiritual care}

Depending from legislation and hospital rules, patients affected from COVID-19 are allowed to receive visitors only to a very limited extent or not at all. This is particularly burdensome for both patients and their loved ones. Protection of the public must be weighted against the patient's and the family's suffering. Within the legal framework, the proportionality of measures should be assessed and discussed, especially in the case of a dying patient.

To ease the burden of isolation, suitable tools for electronic communication should be provided whenever possible. Simple measures like the provision of free telephone cards (some patients do not bring a suitable charging cable for their mobile) may be helpful. Generally, psychosocial and spiritual care of patients, relatives, and healthcare professionals are of paramount importance (Borasio et al., 2020).

\section{Allocation of personnel resources}

To minimize infection risk and to optimize the allocation of personnel resources, it may be advisable for multiprofessional HPCTs to develop pandemic-specific care strategies. Fausto et al. (2020) suggest to distinguish between "conventional", "contingency," and "crisis" care. While "contingency" care is able to offer functionally equivalent care during pandemic, in "crisis care" resources are inadequate and efforts should be made to provide care to the highest level possible. The authors suggest to develop separate strategies for each scenario. To build further capacity, the time between two waves of the pandemic should be used to provide focused education on end-of-life care for patients with COVID-19 to other healthcare professionals such as nurses and emergency department staff (Arya et al., 2020).

\section{Conclusions}

It is mandatory that HPCTs take responsibility for the care of dying COVID patients and their families. To provide palliative care to the highest level possible, HPCTs should share experiences from the past weeks and prepare for the challenges we will face during the next expected pandemic outbreaks.

Acknowledgments. I thank Gian Domenico Borasio for his helpful comments.

Funding. This research received no specific grant from any funding agencies and commercial or not-for-profit sectors.

Conflict of interest. There are no conflicts of interests.

\section{References}

Arya A, Buchman S, Gagnon B, et al. (2020) Pandemic palliative care: Beyond ventilators and saving lives. Canadian Medical Association Journal 192(15), E400-E404. doi:10.1503/cmaj.200465.

Borasio GD, Gamondi C, Obrist M, et al. (2020) For the Covid-task force of palliative Ch. COVID-19: Decision making and palliative care. Swiss Medical Weekly. doi:10.4414/smw.2020.20233.

Downar J, Seccareccia D and Associated Medical Services Inc. Educational Fellows in Care at the End of Life (2010) Palliating a pandemic: "All 
patients must be cared for". Journal of Pain and Symptom Management 39 (2), 291-295. doi:10.1016/j.jpainsymman.2009.11.241.

Emanuel EJ, Persad G, Upshur R, et al. (2020) Fair allocation of scarce medical resources in the time of Covid-19. New England Journal of Medicine. doi:10.1056/NEJMsb2005114.

Fausto J, Hirano L, Lam D, et al. (2020) Creating a palliative care inpatient response plan for COVID-19 - The UW medicine experience. Journal of Pain Symptom Management. doi:10.1016/j.jpainsymman.2020. 03.025 .

Goh KJ, Choong MC, Cheong EH, et al. (2020) Rapid progression to acute respiratory distress syndrome: Review of current understanding of critical illness from COVID-19 infection. Annals of the Academy of Medicine, Singapore 49(1), 1-9.

Kissler SM, Tedijanto C, Goldstein E, et al. (2020) Projecting the transmission dynamics of SARS-CoV-2 through the postpandemic period. Science. doi:10.1126/science.abb5793.

Swiss Academy of Medical Sciences (2020). COVID-19 pandemic: Triage for intensive-care treatment under resource scarcity. Available at: http://www. sams.ch/en/coronavirus.

Truog RD, Mitchell C and Daley GQ (2020) The Toughest Triage Allocating ventilators in a pandemic. New England Journal of Medicine. doi:10.1056/NEJMp2005689. 